\title{
A cluster based object oriented service discovery and advertisement proposed for MANETS
}

\begin{abstract}
A Resource management is one of the important services that are offered in any network. Mobile ad hoc network (MANET) is a gathering of mobile wireless nodes that are located in dynamic network with unnecessary using pre-existing infrastructure all mobile nodes which are free for any movement to any side and they are less network. This network is going ahead to zero configurations that would create some problems in mobile computing. There are variety limitations in Ad hoc network that need to be considered among design and implementation for it. This paper is concerned with the hybridization of object oriented method and agent to manage computing in mobile Ad hoc networks. The proposed approach causes the network load and latency to be decreased. Since the amount of communication messages is a vital issue in MANETs, also a strategy is introduced based on moving service advertisement from application layer to routing layer to reduction of network traffic. It's expected that the proposed resource management method reduces the latency, overhead and energy consumption in MANET.
\end{abstract}

Keyword: MANETs; Mobile agent; Service discovery 